\title{
Re: Role of Tamsulosin, Tadalafil and Silodosin as the Medical Expulsive Therapy in Lower Ureteric Stone: A Randomized Trial (A Pilot Study)
}

\author{
Kumar S, Jayant K, Agrawal MM, Singh SK, Agrawal S, Parmar KM
}

Postgraduate Institute of Medical Education and Research, Department of Urology, Chandigarh, India

Urology 2015;85:59-63.

\section{EDITORIAL COMMENT}

Urolithiasis is a common and increasing urologic problem in Anatolia region of Turkey. This situation necessitates safe and efficacious treatment. Medical expulsive therapy is a proven treatment modality in informed patients according to the guidelines. Alpha blockers and nifedipine are accepted therapeutics while tamsulosin is the most used one. In this study, patients were randomized to one of three groups ( $n=90$, each). Interestingly, one arm was composed of tadalafil. The hypothesis was ureteral relaxation by NO/cGMP pathway. Absence of placebo arm is a negative point for the design of this study. All patients had 5-10 mm stones localized in the distal ureter. They found that silodosin group had significantly higher stone-free rate and shorter stone expulsion time than tamsulosin and tadalafil groups (83\%,64\%, and 66\%, respectively). A brief conclusion of this study indicates that tadalafil was as effective as tamsulosin while silodosin was the most potent one. In the literature, tadalafil and medical expulsive therapy search meets only three studies with this same group. Therefore, it is early to give a comment on this topic, but it would be clarified in near future.

Barbaros Başeskioğlu, MD

\section{Re: Minimally Invasive Treatment of Renal Transplant Nephrolithiasis}

\author{
He-jia Yuan, Dian-dong Yang, Yuan-shan Cui, Chang-ping Men, Zhen-li Gao, L. Shi, Ji-tao Wu \\ Yantai Yuhuangding Hospital Affiliated to Medical College of Qingdao University, Department of Urology, Yantai, China
}

World J Urol 2015; doi: 10.1007/s00345-015-1549-9. [Epub ahead of print]

\section{EDITORIAL COMMENT}

Renal transplant stone disease is a rare complication with a reported incidence ranging between $0.2 \%$ and $6.3 \%$. However, consequences of allograft lithiasis might be serious leading to acute renal failure if obstruction occurs. In this retrospective study, the authors have evaluated the efficacy and safety of the minimally invasive procedures used to manage cases of allograft lithiasis in 19 patients (1.2\%) out of 1615 kidney allograft recipients. Percutaneous nephrolithotomy has been the most frequent choice of treatment modality (31.6\%) besides extracorporeal shock wave lithotripsy, flexible ureterorenoscopy, conservative management or combination of those modalities which have rendered stone-free rates in all patients in this cohort. However, it must be kept in mind that immunosuppressed state, the extra anatomic location of the transplanted kidney and denervation of the allograft can make the diagnosis and management challenging in renal transplant nephrolithiasis.

Y. Kamil Yakupoğlu MD 